\title{
The interaction between duration, velocity and repetitive auditory stimulation
}

\author{
Alexis D.J. Makin *, Ellen Poliakoff, Joe Dillon, Aimee Perrin, Thomas Mullet, Luke A. Jones \\ School of Psychological Sciences, University of Manchester, Oxford Rd, Manchester, M13 9PL, United Kingdom
}

\section{A R T I C L E I N F O}

\section{Article history:}

Received 1 December 2011

Received in revised form 25 January 2012

Accepted 31 January 2012

Available online 26 February 2012

\section{PsycINFO classification:}

2323

2326

2346

Keywords:

Time dilation

Click trains

Scalar expectancy theory

Internal clock

Speed

Slope effect

\begin{abstract}
A B S T R A C T
Repetitive auditory stimulation (with click trains) and visual velocity signals both have intriguing effects on the subjective passage of time. Previous studies have established that prior presentation of auditory clicks increases the subjective duration of subsequent sensory input, and that faster moving stimuli are also judged to have been presented for longer (the time dilation effect). However, the effect of clicks on velocity estimation is unknown, and the nature of the time dilation effect remains ambiguous. Here were present a series of five experiments to explore these phenomena in more detail. Participants viewed a rightward moving grating which traveled at velocities ranging from 5 to $15^{\circ}$ s and which lasted for durations of 500 to $1500 \mathrm{~ms}$. Gratings were preceded by clicks, silence or white noise. It was found that both clicks and higher velocities increased subjective duration. It was also found that the time dilation effect was a constant proportion of stimulus duration. This implies that faster velocity increases the rate of the pacemaker component of the internal clock. Conversely, clicks increased subjective velocity, but the magnitude of this effect was not proportional to actual velocity. Through considerations of these results, we conclude that clicks independently affect velocity and duration representations.
\end{abstract}

(c) 2012 Elsevier B.V. All rights reserved.

\section{Introduction}

Humans can discriminate the duration for which a brief visual stimulus is presented (Wearden, Edwards, Fakhri, \& Percival, 1998). If the stimulus is moving, they can also discriminate its velocity (Makin, Poliakoff, \& El-Deredy, 2009). The current exploratory study aimed to extend the existing literature by clarifying the nature of two factors known to influence temporal judgments, and investigating a further, previously unexplored effect, which we describe below.

\subsection{Known effect 1: auditory click trains increase subjective duration}

It has been demonstrated that the prior presentation of auditory click trains elongates the perceived duration of a subsequent visual stimulus (e.g. Penton-Voak, Edwards, Percival, \& Wearden, 1996; Treisman, Faulkner, Naish, \& Brogan, 1990). These authors theorized that duration estimates are produced by an internal pacemaker-accumulator clock (Church, 1984; Gibbon, 1977; Wearden, 1991). The pacemaker emits pulses or 'ticks', and the accumulator counts the number of ticks. Click trains increase the speed of the pacemaker, and

\footnotetext{
* Corresponding author at: Department of Experimental Psychology, Eleanor Rathbone Building, University of Liverpool, Liverpool, L69 7ZA, United Kingdom. Tel.: +441517942000 .

E-mail addresses: alexis.makin@liverpool.ac.uk (A.D.J. Makin),

ellen.poliakoff@manchester.ac.uk (E. Poliakoff), luke.jones@manchester.ac.uk (L.A. Jones).
}

therefore more ticks accumulate within a given duration, which is consequently judged to have lasted longer. It is important to recognize that this is not intended as a neural model of time perception: The pacemakeraccumulator clock is admittedly metaphorical. However, it provides a useful framework for understanding the various influences on human duration estimation, and we refer to it when explaining our results.

Another important observation made by Penton-Voak et al. (1996) was that the slope of the relationship between real duration and estimated duration is steeper after clicks than after silence. Such a slope effect is exactly what would be expected if the clicks increased the pacemaker rate: If the participant is relying on a 'sped up' pacemaker after a click train, the number of additional ticks accumulated will increase with the actual duration of the stimulus presented (Wearden et al., 1998).

In the current work, we ask whether the well-established effect of click trains on subjective duration applies when people judge the duration of a moving stimulus. Given that motion itself influences perceived duration (as described below), it is uncertain whether these click trains would have the typical effect.

\subsection{Known effect 2: motion increases subjective duration}

In other experiments, it has been found that moving stimuli are typically judged to last longer than static ones of the same duration. This time dilation effect is proportional to stimulus speed; with faster motion judged to be presented for longer than slower motion (Brown, 1995). In a more recent study, Kanai, Paffen, Hogendoorn, 
and Verstraten (2006) asked participants to reproduce the presentation duration of expanding concentric rings by holding down a button. As predicted, the results implied that faster expansions appeared to have lasted longer. It was argued that time is represented as the cumulative number of changes that occur; faster motion causes time dilation because it produces a greater number of changes by the end of the trial. More recently still, this time dilation effect has replicated using a discrimination paradigm (Kaneko \& Murakami, 2009).

\subsection{One unknown effect: do auditory clicks alter the subjective velocity} of moving stimuli?

It is unknown whether auditory click trains systematically alter judgments of velocity. Although click trains could shift subjective velocity in any direction, we predicted that they would increase velocity estimates. This hypothesis is supported by the fact that clicks increase the estimated magnitude of a variety of dynamic stimuli, such as the cumulative length of a series of flashed lines (Droit-Volet, 2010).

\subsection{Current work}

We aimed to explore the effect of auditory click trains on the subjective duration and velocity of moving stimuli. Experiment 1 involved two tasks that addressed these questions in turn, and successfully established that prior presentation of clicks increased duration and velocity estimates, as predicted. However, further experiments were required to adjudicate between various explanations for these effects. In Experiment $2 \mathrm{~A}$ and $\mathrm{B}$, we explored whether the influence of clicks on subjective velocity was due to pre-stimulus alertness or response bias. In Experiment 3, we addressed the same questions with regards to the subjective duration of moving stimuli. Experiment 4 addressed the final unresolved issue; whether time dilation (the effect of velocity on subjective duration) is attributable to response bias.

\section{Experiment 1}

Experiment 1 involved two tasks with identical visual stimuli. Participants observed moving sinusoidal gratings in a square window. The gratings moved rightward at 5,10 or $15^{\circ} / \mathrm{s}$, and were presented for 500, 1000 or $1500 \mathrm{~ms}$. In the Duration Estimation Task (DET), participants estimated the duration of the gratings, while in the Velocity Estimation Task (VET), they estimated grating velocity. In both tasks, gratings were preceded by either an auditory click train or by a silent interval of the same duration. The range of durations was designed to be relatively short in order to prevent participants from chronometric counting (as may happen if longer intervals are used, Wearden et al., 1998). The range of velocities mirrored those used by Kanai et al. (2006).

In the DET, it was predicted that clicks would result in longer duration estimations (Penton-Voak et al., 1996), and that greater stimulus velocity would also have this effect (Brown, 1995). This pattern of results would replicate previous results and also show that the established effect of clicks on subjective duration generalizes to moving stimuli for the first time. The outcome of the VET was not so obvious a priori. It could be that clicks would increase, decrease or simply have no effect on velocity estimates. We considered the first possibility most likely, because, as mentioned, Droit-Volet (2010) recently found that clicks increased the estimated magnitude of various dynamic stimuli (although she did not investigate velocity).

\subsection{Method}

\subsubsection{Participants}

24 undergraduates participated (Mean age $=19.21$ [SD $=1.69$ ]; 2 male). As with all experiments reported here, the study had local ethics committee approval and followed the 1991 Declaration of Helsinki.

\subsubsection{Apparatus}

Participants sat in a darkened room $75 \mathrm{~cm}$ in front of a $40 \mathrm{~cm}$ monitor, which subtended $29.86^{\circ}$ of their visual field. Head position was stabilized with a chin rest, and eye level was always around screen center. They were presented with $11.65^{\circ} \times 11.65^{\circ}$ sinusoidal gratings with a spatial frequency of one cycle per degree. Stimuli were presented using a visual stimulus generator (VISAGE, Cambridge Research Systems). The gratings drifted rightwards at velocities ranging from 4 to $16^{\circ} / \mathrm{s}$. It is important to note that the window in which the gratings were presented did not move. The presentation approximated drifting vertical bars as seen through a window in the center of the screen. Auditory stimulation was presented through headphones. This consisted of $4.5 \mathrm{~s}$ of clicks ( $4 \mathrm{~Hz}$ click train, eighteen individual $125 \mathrm{~ms}$ clicks, $8000 \mathrm{~Hz}$ ) or a click-bounded silence of the same duration. The exact characteristics of the auditory click trains were chosen to match those used in previous cognitive timing studies (e.g. Jones, Allely, \& Wearden, 2011; Penton-Voak et al., 1996).

\subsubsection{Design}

In both tasks, gratings were presented in 18 conditions [ 3 grating velocity $\left(5,10\right.$ or $\left.15^{\circ} / \mathrm{s}\right) \times 3$ grating duration $(500,1000$ or $1500 \mathrm{~ms}) \times 2$ Sound (Clicks, Silence)].

\subsubsection{Procedure}

Participants were presented with 4 blocks of trials. In two of the blocks, they were required to listen to auditory stimulation (clicks or silence) and then estimate the duration of the moving gratings (DET). In the other two blocks, they listened to auditory stimulation (clicks or silence) and then estimated the velocity of the moving gratings (VET, Fig. 1A). It is important to note that the clicks were presented before the visual stimulus in both tasks, so there was no need to divide attention between visual and auditory modalities. Estimates were reported with the number keys on a standard computer keyboard. A prompt screen was shown at the end of each trial, prompting the participant to enter a judgment. In the DET, this screen instructed participants to enter the duration of the moving grating (300-1700 ms). In the VET, it prompted them to enter the velocity of the moving grating (4-16 cycles per second). Estimates outside these ranges were not accepted, and a new screen prompted the participant to re-enter a different response. There was no time limit for responding, and no requirement to maintain ocular fixation.

Both tasks consisted of 90 experimental trials with each of the 18 conditions repeated 5 times. This experiment also included additional 30 'filler trials' in each task, with velocity randomized between 4 and $16^{\circ} / \mathrm{s}$ and duration between 300 and $1700 \mathrm{~ms}$ (with equal number of filler trials proceeded by clicks and silence). These parameters were designed to prevent over-learning of the parameters in the experimental conditions. However, velocity was always $4 \% \mathrm{~s}$ in the filler trials of the DET due to a programming error. Although unfortunate, this problem was probably insignificant, because all the key effects were replicated in the other experiments when it was rectified. The order of presentation of the VET and DET was counterbalanced.

Before the DET, participants viewed examples of the longest and shortest durations and were told the correct response. Likewise, before the VET they were shown the fastest and slowest velocities and told the correct response. Participants also completed 14 trial practice blocks before each task. For the DET, this comprised two repeats of 300, $500700,1000,1300,1500$ and $1700 \mathrm{~ms}$, with velocity at $4^{\circ} / \mathrm{s}$. For the VET practice block, two gratings were presented at velocities ranging from 4 to $16^{\circ} / \mathrm{s}$ in $2 \%$ increments, and duration was randomized between 300 and $1700 \mathrm{~ms}$.

\subsubsection{Analysis}

Mean estimated duration and velocity in each condition were obtained from each participant in the DET and VET respectively. Note 
A
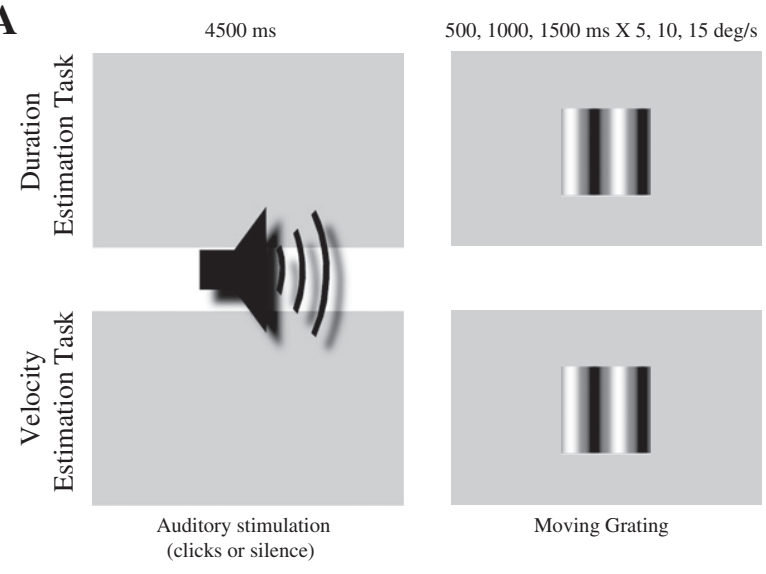

Moving Grating
Until response
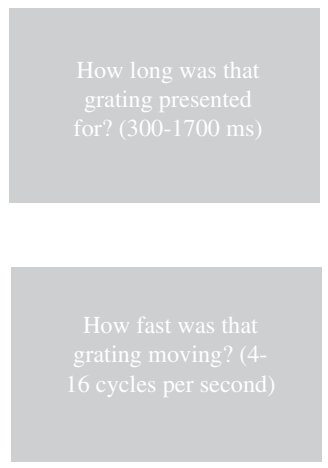

Prompt Screen

B

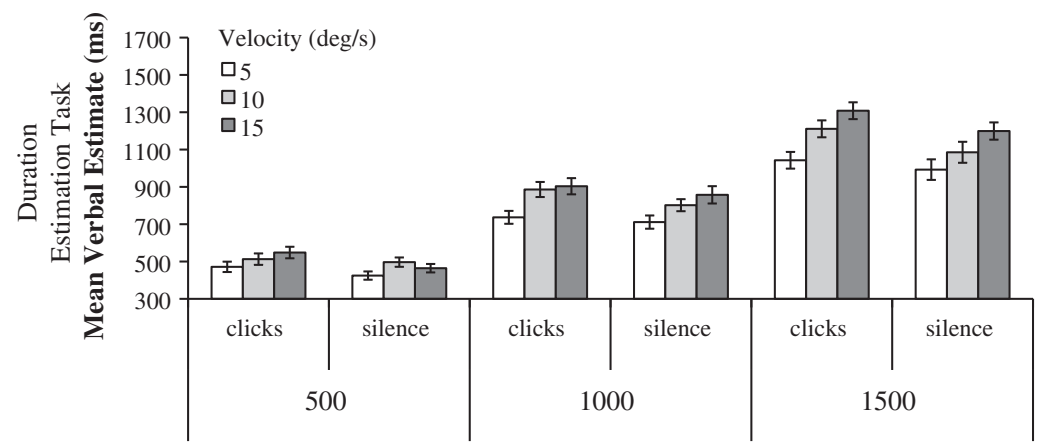

Stimulus Duration (ms)

C

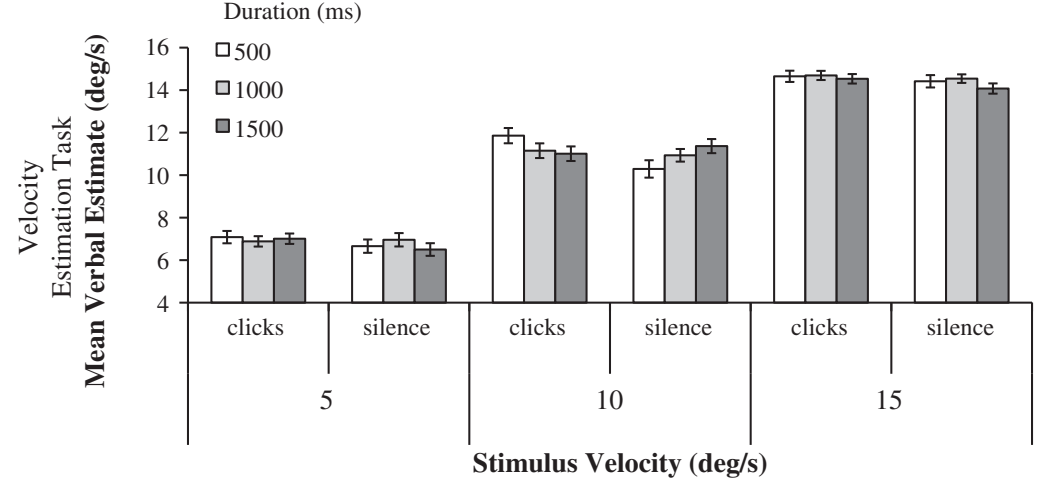

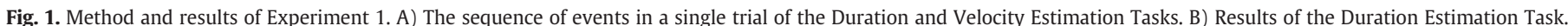

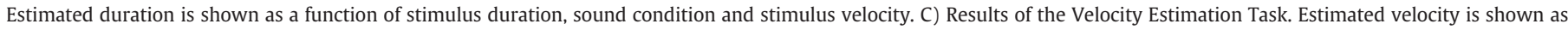
a function of stimulus velocity, auditory stimuli and stimulus duration. Error bars $= \pm 1$ S.E.M.

that filler trials were not analyzed. Mean estimates from experimental trials were analyzed as a function of Velocity $\left(5,10,15^{\circ} / \mathrm{s}\right)$, Duration $(500$, $1000,1500 \mathrm{~ms}$ ) and Sound (Clicks, Silence) with repeated measures ANOVA $(\alpha=0.05)$. Outliers $>2.5$ standard deviations from the condition mean were replaced with the next most extreme value ( 8 data points, $<1 \%$ ). This is a conservative approach to outlier removal: It minimizes alteration to the distribution of values, while also making the mean more representative. The Greenhouse-Geisser correction factor was used when the sphericity assumption was violated. ${ }^{1}$

\footnotetext{
${ }^{1}$ Some variables violated the assumption of normality according to the KolmogorovSmirnov test $(p<0.05 ; 25 \%$ in Experiment $1,9 \%$ in Experiment $2 \mathrm{~A}, 15 \%$ in Experiment 2B, 6\% in Experiment 3 and 21\% in Experiment 4). Although it has been claimed that ANOVA is robust to violations of the normality assumption (Glass, Peckham, \& Sanders, 1972), this issue is still controversial (Erceg-Hurn \& Mirosevic, 2008). However, we are confident our conclusions are valid because all the main effects could be replicated with non-parametric tests (Wilcoxon signed ranks and Friedman ANOVAs, $p<0.05$ ).
}

\subsection{Results}

The results of the DET are shown in Fig. 1B. Inspection of the figures suggests that participants estimated gratings as lasting longer when gratings were either of a longer duration, preceded by clicks, or faster. The results of the VET are shown in Fig. 1C. Gratings were judged faster when they actually were faster or were preceded by clicks. Both tasks were analyzed using 3 factor repeated measures ANOVAs [3 Duration $(500,1000$ or $1500 \mathrm{~ms}) \times 2$ Sound (Clicks, Silence $) \times 3$ Velocity $(5,10$ or $\left.15^{\circ} / \mathrm{s}\right)$ ].

\subsubsection{Duration Estimation Task}

There was a main effect of Duration, indicating that participants could reliably distinguish between 500,1000 and 1500 ms presentations $(F(1.29,29.76)=324.59, p<.001)$. Participants estimated durations as longer after clicks than silence $(F(1,23)=10.75, p=.003)$, and faster gratings as longer $(F(1.54,35.51)=33.79, p<.001)$. The 
Duration $\times$ Velocity interaction was also significant, $F(2.79,64.26)=$ $6.78, p=.001$, because the effect of Velocity on duration estimates was greater at longer durations. This was confirmed by post-hoc tests showing that the mean difference between 5 and $15 \%$ s conditions was significantly greater in the $1000 \mathrm{~ms}$ condition than the $500 \mathrm{~ms}$ condition ( $154 \mathrm{vs} .58 \mathrm{~ms}, t(23)=3.414, p=.002$ ), and marginally greater in the $1500 \mathrm{~ms}$ than the $1000 \mathrm{~ms}$ condition, (236 vs. $154 \mathrm{~ms}, t(23)=1.921 p=.067$, Fig. 1B). No other interactions were significant (highest $F(1.83,42.04)=2.49, p=.10)$.

\subsubsection{Velocity Estimation Task}

Participants could distinguish between 5,10 and $15^{\circ} / \mathrm{s}$ gratings $(F(1.55,35.68)=622.99, p<.001$, Fig. $1 C)$. They also reported gratings as faster after clicks than after silence $(F(1,23)=15.10, p=.001)$. There was no main effect of Duration on velocity estimation $(F(1.32$, $30.31)<1$, NS), and no Duration $\times$ Velocity interaction ( $F(3.19$, $73.35)<1$, NS). However there was a significant Sound $\times$ Duration interaction $(F(1.94,44.72)=12.37, p<.001)$ and 3 way Velocity $\times$ Sound $\times$ Duration interaction $(F(2.96,68.09)=7.38, p<.001)$. These interactions are difficult to interpret theoretically and are not followed up here for the sake of brevity.

\subsection{Discussion}

The key findings in Experiment 1 were as follows. First, in the DET, participants estimated moving gratings to have been presented for longer when this was the case, when they were faster or when they were preceded by clicks. These effects replicate previous findings (Kanai et al., 2006; Penton-Voak et al., 1996), which increases confidence in our experimental set up. It also confirms that clicks can affect the perceived duration of moving stimuli. We consider this novel result further in Experiment 3. It was also found that the effect of velocity increased with the duration to be judged. This replicates the results of Kanai et al. (2006), but with a verbal estimation rather than a temporal production task. We address this further in Experiment 4.

The most important novel finding of Experiment 1 was that participants judged stimuli as faster when they were preceded by clicks. However, it is also possible that the effect is not unique to clicks, but would be produced by any salient auditory stimulation that makes participants more alert during the pre-stimulus period. While it is known that white noise does not prolong subjective duration, implying that there is something special about repetitive stimulation (Jones et al., 2011), this remains to be established in the context of velocity estimation. This issue was addressed in Experiment 2A. A second concern was that the VET in Experiment 1 cannot rule out the possibility that clicks simply biased the participants towards producing higher velocity estimates, without altering velocity perception per se. In other words, the clicks could have distorted decision making, rather than velocity representations. This was explored in Experiment 2B.

\section{Experiment 2}

Experiment 2A was a modified version of the VET from Experiment 1 . However, eleven levels of velocity were included (5 to $15^{\circ} / \mathrm{s}$, in $1 \%$ increments) presented for the same duration, and we included a white noise condition as well as the original clicks and silence conditions. This allowed us to ascertain whether the effect of clicks on velocity was unique to repetitive stimulation.

\subsection{Method}

A different set of 24 undergraduates participated (Mean age $=19.75$ [SD $=2.75]$; 3 male). The stimulus and apparatus were identical to the VET in Experiment 1, with the exception that there was now a white noise condition and all gratings were presented for one second. The white noise was the same duration as the clicks and silence. Again, participants typed their responses on a computer keyboard at the end of every trial.

There were 33 conditions [3 Sound (Clicks, Silence, White noise) $\times 11$ Velocity ( 5 to $15^{\circ} / \mathrm{s}$ in $1 \%$ s increments) . Each condition was repeated 5 times. There were 30 additional filler trials in which velocity was randomized between 4 and $16^{\circ} / \mathrm{s}$, which were not subsequently analyzed. There were an equal number of clicks, silence and white noise filler trials. Trials were presented in one of four different randomized sequences and 6 participants were presented with each trial sequence.

Before the experiment began, participants were shown examples of the fastest and slowest velocities, and were given an 11 trial practice block, in which velocity was again randomized between 4 and $16^{\circ} / \mathrm{s}$ on each trial.

\subsection{Results}

Data were treated in the same way as Experiment 1 . Mean velocity estimates in each condition were obtained from each participant and analyzed with a two-factor repeated measures ANOVA [3 Sound (Clicks, Silence, White noise) $\times 11$ Velocity ( 5 to $15^{\circ} / \mathrm{s}$ in $1 \%$ increments)]. Again a small number of outliers $>2.5$ SD from the condition mean were excluded and replaced with the next most extreme value (1.6\%).

This analysis revealed a main effect of grating Velocity ( $F$ (2.629, $60.477)=317.739, p<.001$ ), confirming that participants were sensitive to this factor (Fig. 2A). There was a main effect of Sound ( $F$ ( 2 , $46)=22.213, p<.001$ ). This was because participants were more likely to report gratings as faster after clicks than either silence or white noise (statistical confirmation is shown in Fig. 2B). There was no Sound $\times$ Velocity interaction $(F(8.93,205.393)=1.188, p=.304)$. This null result implies that the effect of clicks was constant across velocities.

\subsection{Experiment $2 B$}

In Experiment 2A, it was found that clicks produced higher velocity estimates than silence or white noise. This confirms that the effect was not just produced by any auditory stimulus. It could be that the clicks somehow biased participants towards producing higher estimates, without actually affecting velocity perception.

Experiment 2B tested this hypothesis on another set of 24 participants (Mean age $=24.86$ [SD $=5.21]$; 10 male). Outlier replacement rate was $1.1 \%$. Experiment 2B was identical to Experiment 2A, except that the response screen was changed to read, ' $16=$ VERY SLOW to $4=$ NOT VERY SLOW'. Now participants entered a high number to report slower velocities. If clicks simply biased our participants towards entering high numbers, then clicks should lead participants to report slower motion in Experiment 2B. However, if clicks distort subjective velocity then they should again lead to participants reporting faster motion.

The second pattern was obtained. There was a main effect of Sound $(F(2,46)=7.570, p=.001)$ because participants reported faster motion after clicks than either silence or white noise (Fig. $2 \mathrm{C}$ and D). There was also a main effect of Velocity $(F(2.450,56.345)=380.764$, $p<.001$ ), and, like Experiment $2 \mathrm{~A}$, there was no Sound $\times$ Velocity interaction $(F(8.737,200.961)=1.200, p=.298)$.

\subsection{Discussion}

The results of Experiment 2A show that prior presentation of auditory clicks increases subjective velocity by approximately $0.4^{\circ} / \mathrm{s}$ across the range of stimulus velocities presented. However, the effect of click trains on subjective velocity is not produced simply by biasing participants towards the higher end of the response scale, as the same 


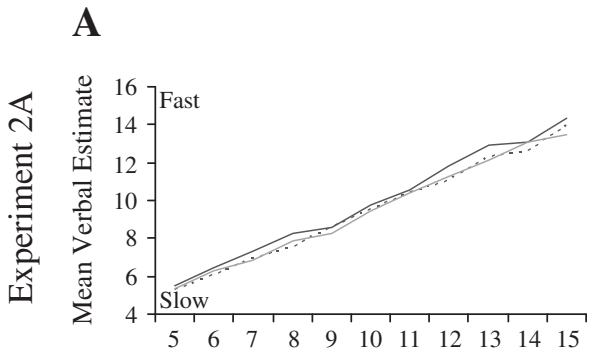

C

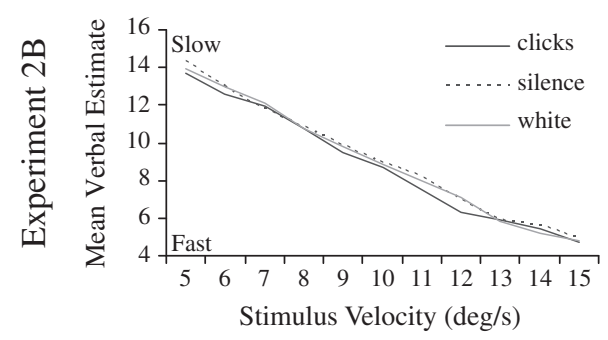

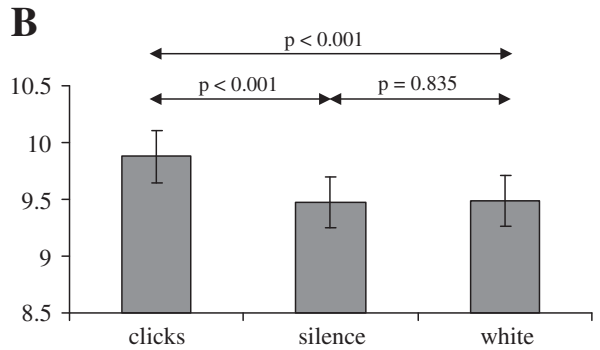

D

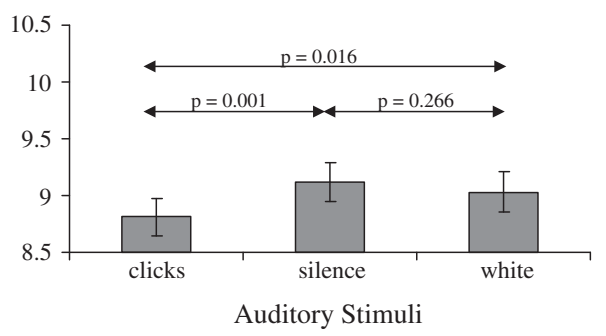

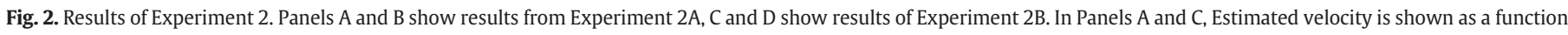

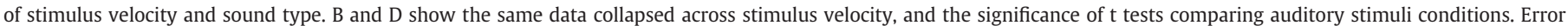
bars $= \pm 1$ S.E.M.

effect was found when the response scale was reversed in Experiment 2B. Nor was the effect germane to all auditory stimulation: there was no difference between white noise and silence conditions. This suggests that the effect of clicks was not produced by simply making the participants more alert, or by virtue of the fact that the prestimulus period was a filled duration. These results add another perceptual judgment to the list of non-temporal judgments that are influenced by auditory click trains (Droit-Volet, 2010; Jones et al., 2011).

\section{Experiment 3}

The DET of Experiment 1 showed that clicks increase subjective duration of moving stimuli. This has been observed before when participants estimate the duration of static stimuli, but not with moving stimuli. There are two subtleties of the effect of click trains, which require further consideration. First, as mentioned above, the effect of clicks is unique; white noise does not increase subjective duration in the same way. Second, the effect of clicks is multiplicative rather than additive: that is, clicks add a constant proportion to subjective duration. For the purpose of illustration, imagine that click trains increase duration estimates by $10 \%$ : An estimate of $100 \mathrm{~ms}$ would increase to $110 \mathrm{~ms}$ after clicks, while an estimate of $1000 \mathrm{~ms}$ would increase to $1100 \mathrm{~ms}$. This results could be termed a 'slope effect' because the slope of the relationship between actual duration and estimated duration is steeper when the stimulus follows the presentation of click trains (Wearden et al., 1998). Experiment 3 asked whether these effects could be generalized to the case where participants estimate the duration of moving stimuli.

Experiment 3 was based on the DET of Experiment 1. However we included eleven levels of the factor Duration (500 to $1500 \mathrm{~ms}$ in $100 \mathrm{~ms}$ increments), and added a white noise condition. Experiment 3 was designed to ascertain whether the effect of clicks increases with duration, and whether it is unique to repetitive stimulation. However, since Experiment 3 was not designed to measure the effect of velocity on subject duration, all gratings were presented at $10^{\circ} / \mathrm{s}$.

\subsection{Method}

A new set of 24 participants was involved (Mean age $=20.96$ [SD = 3.11]; 6 male). Apparatus and stimuli were similar to the DET in Experiment 1, except that velocity was kept at $10^{\circ} / \mathrm{s}$ across conditions. There were 33 conditions [3 Sound (clicks, silence, white noise) $\times 11$ Duration (500 to $1500 \mathrm{~ms}$ in $100 \mathrm{~ms}$ increments)] that were each repeated 5 times. There were 30 filler trials, where the duration was randomized between 300 and 1700 ms, with equal numbers of each sound condition. Again, participants were presented with demonstration trials of the shortest and longest duration, and an 11 trial practice block, where duration was randomized, before the experiment proper.

Statistically, the presence of slope effects can be confirmed in two ways. First, there should be an interaction between Sound and Duration, and this interaction should be produced by an increase in the magnitude of the effect at longer durations. Second, one can obtain the slope of the real duration versus estimated duration relationship for each participant with linear regression, then test whether slope values are significantly greater in the expected condition (cf. Wearden et al., 1998).

\subsection{Results}

Data were processed in the same way as Experiment 1. Mean duration estimates in each condition were obtained from each participant and analyzed with two factor repeated measures ANOVA [3 Sound (Clicks, Silence, White noise) $\times 11$ Duration (500 to $1500 \mathrm{~ms}$ in $100 \mathrm{~ms}$ increments)]. Again, a small number of outliers (>2.5 SD from the condition mean) were excluded and replaced with the next most extreme value $(0.5 \%)$.

Results are shown in Fig. 3. There was a main effect of Duration $(F(1.865,42.904)=120.813, p<.001)$, confirming that participants were performing the task accurately. There was also a main effect of Sound $(F(2,46)=11.335, p<.001)$; participants reported shorter durations in the silence condition than either the white noise or clicks conditions (statistical confirmation is shown in Fig. 3B). There was, 

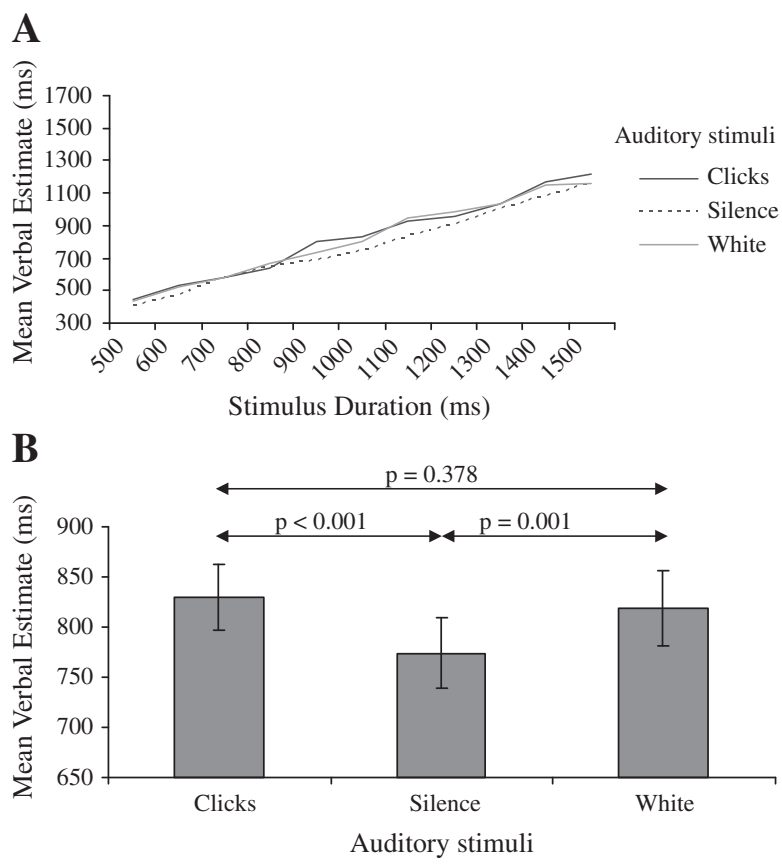

Fig. 3. Results of Experiment 3. A) Estimated duration as a function of actual duration and auditory stimuli. B) The same data collapsed across stimulus duration, and the significance of $t$ tests comparing auditory stimuli conditions. Error bars $= \pm 1$ S.E.M.

however, no Sound $\times$ Duration interaction $(F(9.493,218.335)=$ $1.618, p=.107)$.

There is some suggestion in Fig. 3A that the difference between the Silence condition and the other conditions increased with stimulus duration. Indeed, when clicks and silence are analyzed alone, there was a Sound $\times$ Duration interaction $(F(10,230)=2.10$, $p=.025)$. However, post hoc comparisons between clicks and silence conditions did not support this impression (there were differences at $500,600,900,10001100$ and 1300 ms conditions $(t(23)>2.289$, $p<.033)$, but not at the other levels, $(t(23)<1.732, p>.091)$. Furthermore, mean slope values for the duration vs. estimated duration relationship did not differ between the sound conditions; Clicks $=0.77$, Silence $=0.74$, White Noise $=0.76(F(2,46)<1$, NS $)$.

\subsection{Discussion}

The results of Experiment 3 were unexpected in two respects. First, white noise and clicks produced a similar increase in estimated duration. Second, evidence for slope effects was very limited. It therefore seems possible that the well-established influence of click trains on duration estimation is altered when the stimulus is moving. Both effects will be discussed further in the General discussion.

\section{Experiment 4}

The DET task in Experiment 1 suggested that the effect of velocity on subjective duration was magnified at longer durations. This suggests a slope effect, although the fact that there were only three levels of the dependent variable demands caution. Experiment 4 expands this investigation by presenting more levels of the factor Duration (500 to $1500 \mathrm{~ms}$ in $100 \mathrm{~ms}$ increments). Experiment 4 was not designed to measure the effect of auditory input, so every grating was preceded by silence.

\subsection{Method}

Another set of 24 participants was involved (Mean age $=21.75$ $[\mathrm{SD}=4.26] ; 6$ male). Apparatus were identical to Experiment 3. The design was also similar to Experiment 3, with the exception that all gratings were preceded by silence, and velocity was 5,10 or $15^{\circ} / \mathrm{s}$. There were 33 conditions [3 Velocity $\left(5,10\right.$ or $\left.15^{\circ} / \mathrm{s}\right) \times 11$ Duration (500 to $1500^{\circ} / \mathrm{s}$ in $100 \mathrm{~ms}$ increments)] which were each repeated 5 times. Filler trials, demonstration trials and the practice block were identical to Experiment 3, except that Velocity was 5,10 or $15^{\circ} / \mathrm{s}$ and all trials were preceded by silence.

\subsection{Results}

Data were processed in the same way as Experiment 1. Mean duration estimates in each condition were obtained from each participant and analyzed with two-factor repeated measures ANOVA [3 Velocity $\left(5,10,15^{\circ} / \mathrm{s}\right) \times 11$ Duration ( 500 to $1500 \mathrm{~ms}$ in $100 \mathrm{~ms}$ increments)]. Again, a small number of outliers $>2.5 \mathrm{SD}$ from the condition mean were excluded and replaced with the next highest value (1.13\%).

Results are shown in Fig. 4A. It can be seen that the effect of stimulus velocity on estimated duration increases with stimulus duration. This pattern was confirmed statistically. The main effects of Velocity and Duration, and Velocity $\times$ Duration interaction, were all significant (lowest $F(7.192,165.417)=5.276, p<.001)$. The main effect Velocity was produced by significant differences between each condition (see statistical confirmation in Fig. 4B).

Analysis of slope values revealed that the relationship between duration and estimated duration was steepest when the stimulus was moving at $15^{\circ} / \mathrm{s}$, (Mean slope value $=0.80$ ), less steep at $10^{\circ} / \mathrm{s}$ $(\mathrm{M}=0.67)$ and then shallowest at $5^{\circ} / \mathrm{s}, \quad(\mathrm{M}=0.57),(F \quad(1.291$, $29.686)=12.05, p=.001)$. Post hoc tests revealed that slope values differed between all these levels, $(t(23)>2.555 p<.018)$. From this data set, it can be concluded that a $5 \%$ s increase in stimulus velocity produces an increase in subjective duration of approximately $9 \%$.

\subsection{Discussion}

Experiment 4 provided clear evidence that time dilation affects the slope of the duration vs. estimated duration relationship. Faster stimuli were judged as having lasted longer, and this was effect was a constant proportion of stimulus duration. These slope effects in Experiment 4 were far more robust than any slope effect associated with clicks in Experiment 3 and contrast with the additive effects of Experiment 2, where clicks were found to increase subjective velocity by a constant value.

\section{General discussion}

This series of experiments examined the relationship between cognitive representations of duration and velocity, and the influence of auditory click trains. We explored some open questions in the current literature. The three most important empirical findings of this article are discussed in separate sections below.

\subsection{Auditory click trains increase subjective velocity}

The first novel finding was that click trains increased mean velocity estimates. This was found in the Velocity Estimation Task of Experiment 1. Experiment 2A expanded the results of Experiment 1 by demonstrating that the effect of clicks on subjective velocity was unique; white noise did not have a comparable effect. Moreover, the result cannot be attributed to a simple form of response bias in which clicks somehow inclined participants towards selecting higher values on the response scale; participants still estimated gratings as faster after clicks when the response scale was reversed in Experiment 2B.

How, then, do auditory clicks influence subjective velocity? Simple explanations may cite increased attention or alertness. Indeed, 

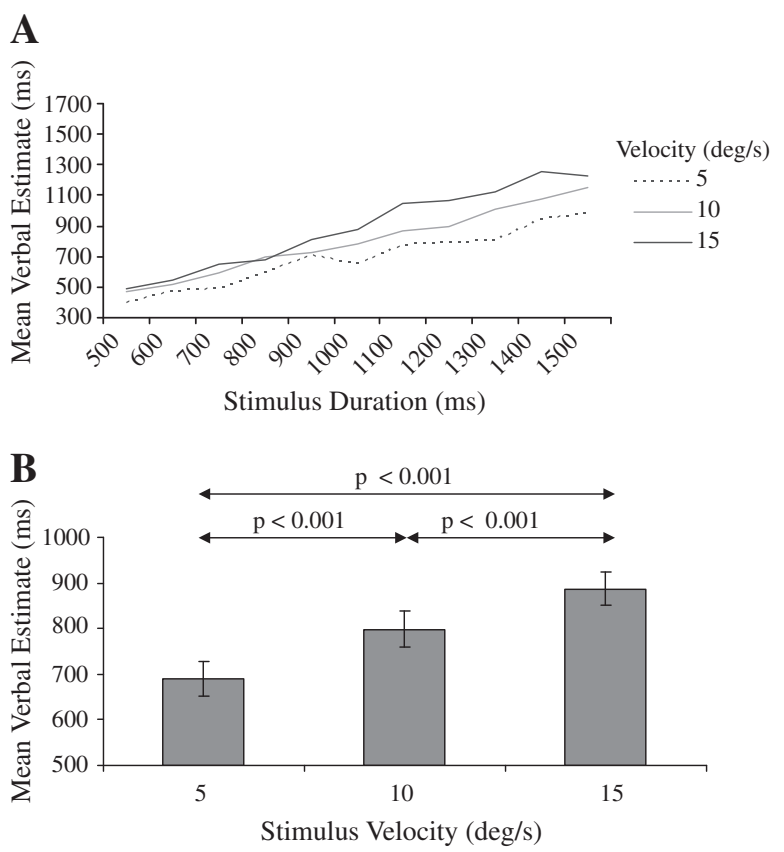

Fig. 4. Results of Experiment 4. A) Estimated duration as a function of stimulus duration and velocity. B) The same data collapsed across stimulus duration and the significance of $t$ tests comparing stimulus velocity conditions. Error bars $= \pm 1$ S.E.M.

previous studies have found that attention makes moving objects appear faster (Turatto, Vescovi, \& Valsecchi, 2007). However such accounts would find it difficult to explain the difference between clicks and white noise conditions, as both would presumably increase pre-stimulus alertness. Neural models may provide some insight here. It is possible that perceived velocity is proportional to global excitation in area MT (Medial Temporal, Krekelberg, van Wezel, \& Albright, 2006). Moreover the auditory click trains could have produced pre-stimulus brain oscillations: a manipulation known to enhance subsequent processing (Hanslmayr et al., 2005). This speculative account could be tested further by systematically manipulating the frequency of auditory click trains, and the temporal or spatial frequency of moving gratings.

The effect of clicks on subjective velocity may be surprising if we consider the following: If velocity estimates were produced by calculating the time it took for a target to travel over a given distance, then increasing estimated duration would decrease estimated velocity ( speed $=$ distance/time). We know that clicks increase subjective duration, so, perhaps they should reduce subjective velocity? The fact that we obtained the opposite result implies that clicks independently affect velocity and duration estimations, rather than affecting velocity estimation via their effect on duration.

We did not require our participants to fixate during this experiment, so it is possible that they tracked the moving bars with their eyes. This would have reduced retinal motion signals from the gratings, possibly to zero in the case of perfect ocular pursuit. Drift velocity could nevertheless be estimated from counter-motion of the visual periphery, or efference copy of the oculomotor commands (Leigh \& Zee, 2006). In other words, our stimuli probably produced multiple velocity representations, both sensory and motor. We do not know which of these were affected by prior presentation of auditory click trains.

Leaving these issues to one side, the finding that prior presentation of click trains increases the subjective velocity adds to the body of literature showing that click trains alter a variety of perceptual mechanisms, and not just subjective duration (Droit-Volet, 2010; Jones et al., 2011).
6.2. The specific effect of auditory clicks on subjective duration does not apply to moving stimuli

In Experiment 3, it was found that compared to silence, both clicks and white noise significantly prolonged subjective duration. This result was not predicted because no previous work has found any influence of white noise upon duration estimation (Jones et al., 2011). Another unexpected finding was that the slope values in all auditory stimulation conditions were similar. This contrasts with previous work, where prior presentation of auditory clicks, but not white noise, reliably increases subjective duration by a stable proportion of actual duration (e.g. Penton-Voak et al., 1996).

How can these unusual details be explained? Wearden et al. (1998) proposed two different ways in which clicks could affect an internal pacemaker-accumulator clock and consequently elevate subjective duration. First, clicks could increase pacemaker speed, resulting in a slope effect. Second, they could alter the onset or offset of the transfer of ticks from the pacemaker to the accumulator. For example, if clicks made participants more alert, the transfer of ticks may begin with reduced latency. This would increase the number of accumulated ticks (and thus subjective duration), but without producing a slope effect. Importantly, white noise may also increase alertness, with similar results. Considering these ideas, it seems possible that stimulus motion masked the effect of clicks on the pacemaker (reducing slope effects), but left the more modest influence of increased alertness (which can be produced by either clicks or white noise) intact. Indeed, velocity signals alter subjective duration, probably by altering pacemaker rate (see Experiment 4 and below). Therefore, any changes in pacemaker rate resulting from the prior presentation of clicks would be overwritten by subsequent velocity signals.

\subsection{Time dilation is proportional to duration}

This work reexamined the time dilation effect, where faster moving stimuli appear to last longer. Time dilation manifested as a slope effect: that is, the slope of the actual vs. estimated duration function was steeper when the targets moved faster. This was apparent in the Duration Estimation Task of Experiment 1, and even more clearly in Experiment 4. This replicates the results of Kanai et al. (2006) with a verbal estimation task rather than a temporal production task. If one accepts the validity of the pacemaker-accumulator clock model (described in the Introduction), this pattern can be best explained with reference to an internal pacemaker that runs at a higher rate when stimuli move faster. Indeed, it is perhaps instructive to consider that moving gratings are comparable to click trains, in that they constitute a form of repetitive sensory stimulation.

There are, however, alternative accounts of cognitive timing, which can make sense of this result. For example, the slope effects in Experiment 4 are also consistent with a mechanism that tracks the number of stimulus changes (as proposed by Kanai et al., 2006). Moreover, Penney, Gibbon, and Meck (2000) and Zakay and Block (1998) both proposed models of an internal clock that slightly differ from that described in the Introduction. They suggest that attention modulates the flow of ticks from the pacemaker to the accumulator, implying that if more attention is paid to faster stimuli then this will result in a slope effect.

Finally, we note that the current results only apply to lateral motion, across the fronto-parallel plane. A recent study by Van Wassenhove, Buonomano, Shimojo, and Shams (2008) found that subjective duration increases or decreases depending on whether the stimulus is looming or receding, and that these effects are modulated by the distribution of motion types in the array. There was also some similarity between auditory and visual motion in this study. This strongly suggests that our results would not generalize to all types of motion pattern. However, our results, along with others, 
suggest that stimulus movement predictably alters its estimated duration.

\section{Conclusions}

In summary, this series of experiments produced three novel results, which extend the empirical literature and inform current theories. First, it was found for the first time that click trains increase velocity estimates. This contributes to the literature showing that clicks effect a whole range of perceptual judgments, not just temporal judgments, as previously assumed. The exact mechanism by which auditory click trains alter perception is unknown, but the generic nature of this effect suggests that any insights will have broad implications. Second, it was found that clicks increase the estimated duration of moving stimuli, but only by enhancing pre-stimulus alertness. Thus, stimulus motion may override the well-known influence of clicks on pacemaker rate. Thirdly, faster motion was judged as having lasted longer, and this effect was proportional to duration. This time dilation effect is better explained by velocity signals increasing pacemaker speed than by response bias. We hope our results will be instructive for future researchers interested in these topics.

\section{Acknowledgments}

We would like to thank Alex Leuder for collecting the data for Experiment 2B. We would also like to thank the Economic and Social Sciences Research Council for funding Alexis Makin during the project.

\section{References}

Brown, S. (1995). Time, change, and motion: The effects of stimulus movement on temporal perception. Perception \& Psychophysics, 57, 105-116.

Church, R. M. (1984). Properties of the internal clock. Annals of the New York Academy of Sciences, 423, 566-582.

Droit-Volet, S. (2010). Speeding up a master clock common to time, number and length? Behavioural Processes, 85, 126-134.

Erceg-Hurn, D. M., \& Mirosevic, V. M. (2008). Modern robust statistical methods: An easy way to maximize the accuracy and power of your research. American Psychologist, 7, 591-601.
Gibbon, J. (1977). Scalar expectancy-theory and Weber's law in animal timing. Psychological Review, 84, 279-325.

Glass, G., Peckham, P., \& Sanders, J. (1972). Consequences of failure to meet assumptions underlying the fixed effects analysis of variance and covariance. Review of Educational Research, 42, 237-288.

Hanslmayr, S., Klimesch, W., Sauseng, P., Gruber, W., Doppelmayr, M., Freunberger, R., et al. (2005). Visual discrimination performance is related to decreased alpha amplitude but increased phase locking. Neuroscience Letters, 375, 64-68.

Jones, L. A., Allely, C. S., \& Wearden, J. H. (2011). Click trains and the rate of information processing: Does "speeding up" subjective time make other psychological processes run faster? Quarterly Journal of Experimental Psychology, 64, 363-380.

Kanai, R., Paffen, C. L. E., Hogendoorn, H., \& Verstraten, F. A. J. (2006). Time dilation in dynamic visual display. Journal of Vision, 6, 1421-1430.

Kaneko, S., \& Murakami, I. (2009). Perceived duration of visual motion increases with speed. Journal of Vision, 9, 12.

Krekelberg, B., van Wezel, R. J. A., \& Albright, T. D. (2006). Interactions between speed and contrast tuning in the middle temporal area: Implications for the neural code for speed. Journal of Neuroscience, 26, 8988-8998.

Leigh, R., \& Zee, D. S. (2006). Smooth pursuit and visual fixation. The neurology of eye movements (4th Edition). Chapter 4.

Makin, A. D. J., Poliakoff, E., \& El-Deredy, W. (2009). Tracking visible and occluded targets: Changes in event related potentials during motion extrapolation. Neuropsychologia, 47, 1128-1137.

Penney, T. B., Gibbon, J., \& Meck, W. H. (2000). Differential effects of auditory and visual signals on clock speed and temporal memory. Journal of Experimental Psychology. Human Perception and Performance, 26, 1770-1787.

Penton-Voak, I. S., Edwards, H., Percival, A., \& Wearden, J. H. (1996). Speeding up an internal clock in humans? Effects of click trains on subjective duration. Journal of Experimental Psychology. Animal Behavior Processes, 22, 307-320.

Treisman, M., Faulkner, A., Naish, P. L. N., \& Brogan, D. (1990). The internal clock: Evidence for a temporal oscillator underlying time perception with some estimates of its characteristic frequency. Perception, 19, 705-748.

Turatto, M., Vescovi, M., \& Valsecchi, M. (2007). Attention makes moving objects be perceived to move faster. Vision Research, 47, 166-178.

Van Wassenhove, V., Buonomano, D. V., Shimojo, S., \& Shams, L. (2008). Distortions of subjective time perception within and across senses. PLoS One, 3, e1437, doi: 10.1371/journal.pone.0001437.

Wearden, J. H. (1991). Do humans possess an internal clock with scalar timing properties. Learning and Motivation, 22, 59-83.

Wearden, J. H., Edwards, H., Fakhri, M., \& Percival, A. (1998). Why "sounds are judged longer than lights": Application of a model of the internal clock in humans. Quarterly Journal of Experimental Psychology Section B-Comparative and Physiological Psychology, $51,97-120$.

Zakay, D., \& Block, R. A. (1998). New perspectives on prospective time estimation. In G. De Keyser, D. Ydewalle, \& A. Vandierendonck (Eds.), Time and dynamic control of behaviour (pp. 129-141). Gottingen: Hogrefe and Huber. 